\title{
Performance Upgradation through Task Allocation of Distributed Networks
}

\author{
Kapil Govil \\ School of Engineering \& Technology, ITM University Gwalior
}

\begin{abstract}
Normally the distributed network has to execute the tasks that shall be the more than the number of processors. The assignment problem is a case of linear programming helps to solve the problems related to tasks and processors. The problem of execution of " $m$ " tasks to " $n$ " processors $(m>n)$ in a distributed networks is addressed here through a new modified tasks allocation policy for distributed networks. The model, presented in this paper allocates the tasks or modules to the processor to increase the performance and to reduce the execution time. This paper reduces the problem of allocation of tasks where number of processors is less than the number of tasks. The example mentioned in the paper has three tasks and solved it in such a way that the task $t_{1}$ processed with minimum time, the task $t_{2}$ with minimum cost while the task $t_{3}$ with maximum reliability. In this problem, the tasks are fused (or clubbed) with another task(s) on the basis of minimum communication cost to form a balanced allocation.
\end{abstract}

\section{Keywords}

Allocation, Cost, Distributed Network, Performance, Processor, Reliability, Task, Time.

\section{INTRODUCTION}

Such type of research problems in which the performance of the distributed systems is to be upgraded, requires either processing time or cost to be minimized or reliability to be maximized by deciding the strategy of allocation of tasks to the processors of the distributed systems. These problems may be categorized as static $(15,16,17,19,29)$ and dynamic $(2$, $14,18,19,26,27)$ in nature. Some of the other related methods have been reported in the literature, such as, Integer programming (7, 23), Branch and Bound technique (28), Matrix Reduction technique $(11,30,31)$, Reliability Optimization $(1,12,20,21,24)$, Load Balancing $(2,9,10)$ and Modeling $(3,6,8)$. The series parallel redundancyallocation problem has been studied with different approaches, such as, Dynamic programming (4, 10, 13), Integer programming $(7,23)$, and Heuristic techniques $(5,22$, 25).

\section{OBJECTIVE}

The objective of the present research paper is to enhance the performance of the distributed systems by using the proper utilization of its processors. A set of tasks have to be processed by the processors of the network, while each of the task have the modules and the number of modules are more than the number of processors of the network. The processing of a task is means that all of its modules get processed. Performance is the measure in term of either time or cost or reliability of the modules of a task that have to process on the processors of the system and these have to be optimally processed i.e., either time or cost to be minimized or reliability to be maximized.

\section{TECHNIQUE}

To evaluate the optimal time or cost or reliability for each task through optimal allocation, initially it has to concentrate on those $(m-n)$ modules that have the highest probability of data transfer with the remaining n modules. Each of these $(m-n)$ modules (say $m_{i k}$ ) of every task is treated as a candidate to be fused with any one (say $m_{i l}$ ) out of the remaining $\mathrm{n}$ modules with which it has the highest communication. Further, all the elements of $k^{\text {th }}$ row and $l^{\text {th }}$ row are to be added in case of time and cost while in case of reliability these rows have to multiply. This will reduces the effectiveness matrix for each task in to a square matrix. Now the problem remains to determine the optimal time or cost or reliability through the allocation strategy by considering either task processing based on time or cost or reliability for all modules to individual processor(s) for each task. For allocation purpose a modified version of row and column assignment method proposed by Kumar et al (17) is employed which allocates all the modules of a task to a processor optimally. The functions for obtaining the overall assignment execution time [Etime], execution cost [Ecost], and execution reliability [Ereliability] are as follows:

$$
\begin{aligned}
& \text { Ptime }=\left[\sum_{i=1}^{n}\left\{\sum_{j=1}^{n} P T_{i j} x_{i j}\right\}\right] \\
& \text { Pcost }=\left[\sum_{i=1}^{n}\left\{\sum_{j=1}^{n} E C_{i j} x_{i j}\right\}\right] \\
& \text { Pr elibility }=\left[\prod_{i=1}^{n}\left\{\sum_{j=1}^{n} E R_{i j} x_{i j}\right\}\right]
\end{aligned}
$$

(3)

Where, $\mathrm{x}_{\mathrm{ij}}=\left\{\begin{array}{l}1, \text { if } i^{\text {th }} \text { task is assigned to } j^{\text {th }} \text { processor } \\ 0, \text { otherwise }\end{array}\right.$

\section{ALGORITHM}

Step 1: Start algo

Step 2: $\quad$ Read the number of tasks in $m$

Step 3: $\quad$ Read the number of processors in $n$

Step 4: $\quad$ For I $=1$ to $n$

Step 5:

$$
\begin{aligned}
\text { For } J=1 & \text { to } \mathrm{m} \\
& \text { Read the value in } \\
& \text { PTM }[\mathrm{I}][\mathrm{J}]
\end{aligned}
$$

Step 7:

Step 8:

Step 9:

Step 10: End of I loop

Step 11: For $\mathrm{I}=1$ to $\mathrm{n}$

Step 12: $\quad$ For $\mathrm{J}=1$ to $\mathrm{n}$ 
Step 13:

Step 14:

Step 15:

Step 16:

Step 17:

Step 18: For $\mathrm{I}=1$ to $\mathrm{n}$

Step 19: $\quad$ For $\mathrm{J}=\mathrm{I}$ to $\mathrm{n}$

Step 20:

Step 21:

If $\mathrm{CM}[\mathrm{I}][\mathrm{J}]==1$ then

Store the value of

$\mathrm{P} 1[\mathrm{I}]$ to 1

Step 22:

Store the value of $\mathrm{P} 2[\mathrm{~J}]$ to 1

Step 23: Calculate MAT $[\mathrm{I}][\mathrm{J}]$

Step 24:

Step 25:

Step 26:

Step 27:

Step 28:

Step 29: For $\mathrm{I}=1$ to $\mathrm{n}$

Step 30:

Step 31:

Step 32:

Step 33:

Step 34: End of I loop

Step 35: For $\mathrm{I}=1$ to $\mathrm{n}$

Step 36:

Step 37:

Step 38

Step 39:

Step 40: End of I loop

Step 41: Count the zero(s) in each row

Step 42: Mark the row(s), which have single zero

Step 43: Mark the column, which have single zero

Step 44: Go to the row(s), which have more than one zero. Now select any one zero and cross the leading zero(s), which are in same row and column

Step 45: Mark the assignments

Step 46: Count the total assignment

Step 47: If total number of assignment < order of matrix

Step 48: $\quad$ Go to Step 52

Step 49: Else

Step 50: $\quad$ Go to Step 59

Step 51: End of if statement

Step 52: Mark the rows for which assignment have not been made

Step 53: Mark column that have zeros in marked rows

Step 54: Mark rows that have assignment in marked column

Step 55: Repeat Step 53 \& Step 54 until chain of marking ends

Step 56:

Draw the minimum number of lines through unmarked rows and marked columns to cover all zeros

Step 57: Select the smallest element of the uncovered elements and replace it by zero. Also add this element to positions at which lines intersect to each other only

Step 58: Go to Step 42

Step 59: State processing time

Step 60: End algo

\section{IMPLEMENTATION}

Consider an example consisting of a set $T=\left\{t_{1}, t_{2}, t_{3}\right\}$ of 3 tasks each of them having sets $\mathrm{M}_{1}=\left\{\mathrm{m}_{11}, \mathrm{~m}_{12}, \mathrm{~m}_{13} \mathrm{~m}_{14}, \mathrm{~m}_{15}\right\}$ of 5 modules, $\mathrm{M}_{2}=\left\{\mathrm{m}_{21}, \mathrm{~m}_{22}, \mathrm{~m}_{23} \mathrm{~m}_{24}\right\}$ of 4 modules and $\mathrm{M}_{3}=$ $\left\{\mathrm{m}_{31}, \mathrm{~m}_{32}, \mathrm{~m}_{33} \mathrm{~m}_{34}, \mathrm{~m}_{35}, \mathrm{~m}_{36}\right\}$ of 6 modules respectively. The three processors are available in the distributed network to process the tasks that are represented by the set $\mathrm{P}=\left\{\mathrm{p}_{1}, \mathrm{p}_{2}\right.$, $\mathrm{p}_{3}$ \}. The processing time (t), cost (c) and reliability (r) of each module of every task on various processors are known and mentioned in the following matrix, namely, $\operatorname{PCTR}($, ): 


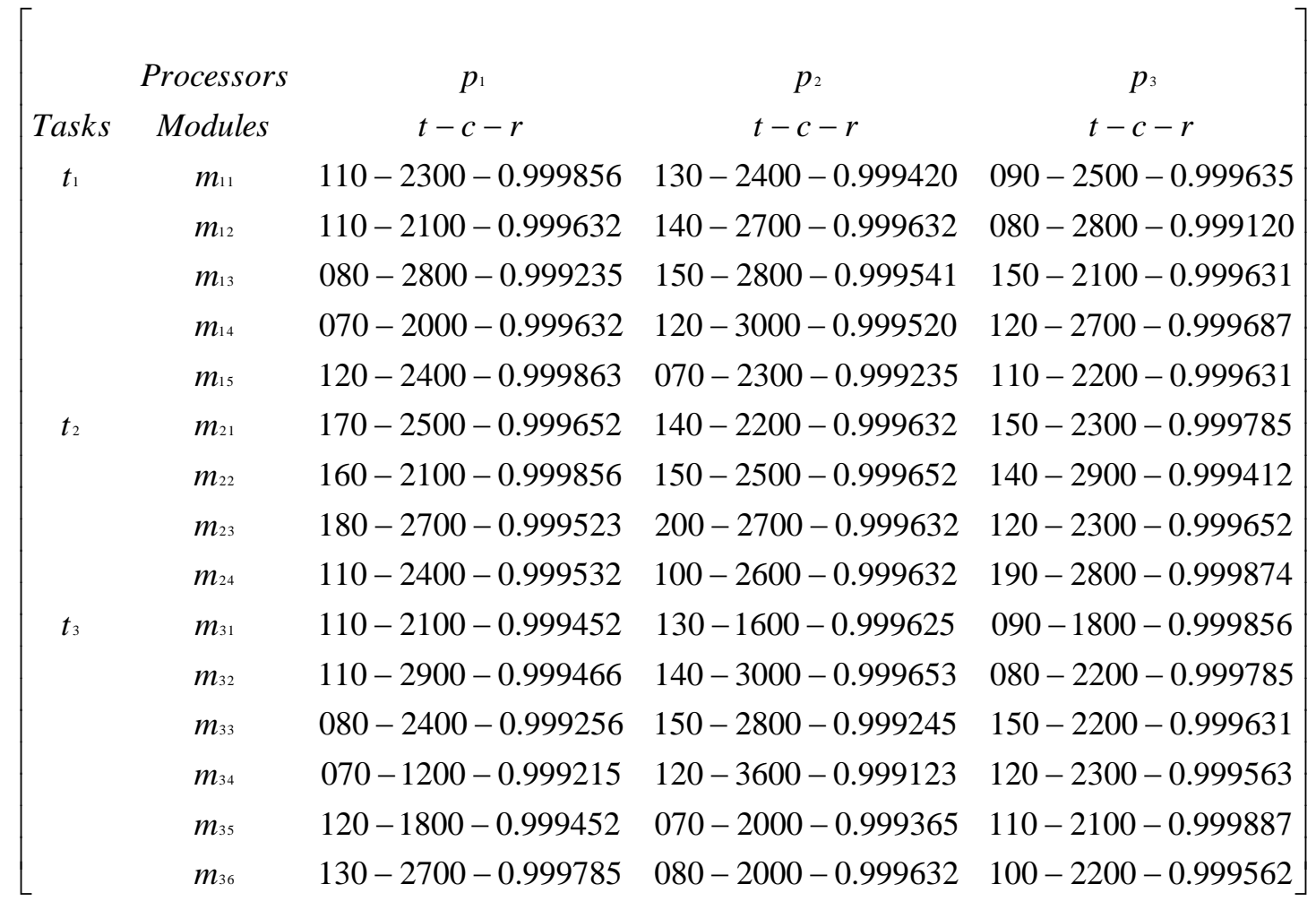

The communication period amongst the modules of each task has also been considered and it is mentioned in the following matrices, namely, $\mathrm{CM}($, ):

For task $t_{l}$, the matrix $\mathrm{CM}(1$,$) is as:$

$\left[\begin{array}{cccccc} & m_{11} & m_{12} & m_{13} & m_{14} & m_{15} \\ m_{11} & 0 & 1 & 6 & 9 & 3 \\ m_{12} & & 0 & 2 & 7 & 8 \\ m_{13} & & & 0 & 4 & 5 \\ m_{14} & & & & 0 & 2 \\ m_{15} & & & & & 0\end{array}\right]$

For task $t_{2}$, the matrix CM $(2$,$) is as:$

$\left[\begin{array}{ccccc} & m_{21} & m_{22} & m_{23} & m_{24} \\ m_{21} & 0 & 2 & 4 & 5 \\ m_{22} & & 0 & 7 & 8 \\ m_{23} & & & 0 & 6 \\ m_{24} & & & & 0\end{array}\right] ;$

For task $t_{3}$, the matrix $\mathrm{CM}(3$, ) is as:

$\left[\begin{array}{ccccccc} & m_{31} & m_{32} & m_{33} & m_{34} & m_{35} & m_{36} \\ m_{31} & 0 & 2 & 3 & 7 & 9 & 6 \\ m_{32} & & 0 & 4 & 8 & 6 & 5 \\ m_{33} & & & 0 & 1 & 5 & 4 \\ m_{34} & & & & 0 & 2 & 3 \\ m_{35} & & & & & 0 & 1 \\ m_{36} & & & & & & 0\end{array}\right]$

Here, it is considered the processing of the tasks $t_{l}$ based on the time constraints (however one may choose the cost or reliability constraints also); $t_{2}$ is based on the cost constraints (however one may choose the time or reliability constraints also); and for the $t_{3}$ it is based on reliability constraints (however one may choose the time or cost constraints also). Further it is also noted that each task has modules that are more than the number of processors in the distributed system. So following data from the matrix PCTR (, ) is used i.e, 


$\begin{array}{ccc} & \text { Processors } & p_{1} \\ \text { Tasks } & \text { Modules } & t-c-r \\ t_{1} & m_{11} & 070-\cdots-\cdots \\ & m_{12} & 080-\cdots-\cdots \\ & m_{13} & 120-\cdots-\cdots \\ & m_{14} & 160-\cdots-\cdots \\ & m_{15} & 170-\cdots-\cdots \\ t_{2} & m_{21} & \cdots-2500-\cdots \\ & m_{22} & \cdots-2100-\cdots \\ & m_{23} & \cdots-2700-\cdots \\ & m_{24} & \cdots-2400-\cdots \\ & m_{31} & \cdots-\cdots-0.999452 \\ & m_{32} & \cdots-\cdots-0.999466 \\ & m_{33} & \cdots-\cdots-0.999256 \\ & m_{34} & \cdots-\cdots-0.999215 \\ & m_{35} & \cdots-\cdots-0.999452 \\ & m_{36} & \cdots-\cdots-0.999785\end{array}$

The task $t_{l}$ has five modules, so that on the basis of highest communication, the modules $m_{11} \& m_{14}$ and $m_{12} \& m_{15}$ are fused together to reduce the effectiveness matrix square. The task $t_{2}$ has four modules, so that on the basis of highest communication, the modules $m_{21} \& m_{24}$ are fused together to

$$
\left[\begin{array}{ccc} 
& \text { Processors } & p_{1} \\
\text { Tasks } & \text { Modules } & t-c-r \\
t_{1} & m_{11} * m_{14} & 230-\cdots-\cdots \\
& m_{12} * m_{15} & 250-\cdots-\cdots \\
& m_{13} & 120-\cdots-\cdots \\
& & \\
t_{2} & m_{21} & \cdots-2500-\cdots \\
& m_{22} * m_{24} & \cdots-4500-\cdots \\
& m_{23} & \cdots-2700-\cdots \\
& & \\
t_{3} & m_{31} * m_{35} & \cdots-\cdots-0.998904 \\
& m_{32} * m_{34} & \cdots-\cdots-0.998681 \\
& m_{33} * m_{36} & \cdots-\cdots-0.999041
\end{array}\right.
$$

The results of the allocations based on time for the task $t_{l}$ are obtained after implementing the row \& column assignment process as suggested by Kumar et al (17), are mentioned below in the Table 1;

Table 1. Time based Allocation for task $\boldsymbol{t}_{\boldsymbol{1}}$

$\begin{array}{ccccc}\text { Modules } & \rightarrow & \text { Processors } & \text { Time } & \text { Etime } \\ m_{11} * m_{14} & \rightarrow & p_{1} & 230 & \\ m_{12} * m_{15} & \rightarrow & p_{2} & 220 & 590 \\ m_{13} & \rightarrow & p_{3} & 140 & \end{array}$

$$
\left.\begin{array}{cc}
p_{2} & p_{3} \\
t-c-r & t-c-r \\
110-\cdots-\cdots & 120-\cdots-\cdots \\
090-\cdots-\cdots & 130-\cdots-\cdots \\
135-\cdots-\cdots & 140-\cdots-\cdots \\
140-\cdots-\cdots & 150-\cdots-\cdots \\
130-\cdots-\cdots & 160-\cdots-\cdots \\
\cdots-2200-\cdots & \cdots-2300-\cdots \\
\cdots-2800-\cdots & \cdots-2900-\cdots \\
\cdots-2700-\cdots & \cdots-2300-\cdots \\
\cdots-2600-\cdots & \cdots-2800-\cdots \\
\cdots-\cdots-0.999625 & \cdots-\cdots-0.999856 \\
\cdots-\cdots-0.999653 & \cdots-\cdots-0.999785 \\
\cdots-\cdots-0.999245 & \cdots-\cdots-0.999631 \\
\cdots-\cdots-0.999123 & \cdots-\cdots-0.999563 \\
\cdots-\cdots-0.999365 & \cdots-\cdots-0.999887 \\
\cdots-\cdots-0.999632 & \cdots-\cdots-0.999562
\end{array}\right]
$$

\begin{tabular}{|c|c|}
\hline$\cdot-0.998990$ & $\cdots-\cdots-0.999743$ \\
\hline$\cdots-\cdots-0.998776$ & $\cdots-\cdots-0.999348$ \\
\hline$\cdot-0.998877$ & -0.999193 \\
\hline
\end{tabular}

reduce the effectiveness matrix square. The task $t_{3}$ has six modules, so that on the basis of highest communication, the modules $m_{31} \& m_{35}, m_{32} \& m_{32}$ and $m_{34} \& m_{36}$ are fused together to reduce the effectiveness matrix square. The resulting matrix is as:

$$
\begin{array}{cc}
p_{2} & p_{3} \\
t-c-r & t-c-r \\
250-\cdots-\cdots & 270-\cdots-\cdots \\
220-\cdots-\cdots & 290-\cdots-\cdots \\
135-\cdots-\cdots & 140-\cdots-\cdots \\
& \\
\cdots-2200-\cdots & \cdots-2300-\cdots \\
\cdots-5400-\cdots & \cdots-5700-\cdots \\
\cdots-2700-\cdots & \cdots-2300-\cdots
\end{array}
$$

The results of the allocations based on cost for the task $t_{2}$ are obtained after implementing the row \& column assignment process as suggested by Kumar et al (17), are mentioned below in the Table 2;

Table 2. Cost based Allocation for task $\boldsymbol{t}_{2}$

$\begin{array}{ccccc}\text { Modules } & \rightarrow & \text { Processors } & \text { Cost } & \text { Ecost } \\ m_{22} * m_{24} & \rightarrow & p_{1} & 2200 & \\ m_{21} & \rightarrow & p_{2} & 4500 & 9000 \\ m_{23} & \rightarrow & p_{3} & 2300 & \end{array}$


The results of the allocations based on reliability for the task $t_{3}$ are obtained after implementing the row \& column assignment process as suggested by Kumar et al (17), are mentioned below in the Table 3;
Table 3. Reliability based Allocation for task $\boldsymbol{t}_{3}$

$\begin{array}{cccc}\text { Modules } & \rightarrow & \text { Processors } & \text { Reliabit } \\ m_{31} * m_{35} & \rightarrow & p_{1} & 0.9989 \\ m_{32} * m_{34} & \rightarrow & p_{2} & 0.9987 \\ m_{33} * m_{36} & \rightarrow & p_{3} & 0.999 \\ & & & \\ & & & \\ \text { cation Table } & & & \\ \text { Optimal } & \text { Optimal } & \text { Optimal } \\ \text { Etime } & \text { Ecost } & \text { Ereliability } \\ 590 & \ldots & \ldots \\ \ldots & 9000 & \ldots \\ \ldots & \ldots & 0.9966876\end{array}$

\section{CONCLUSION}

This paper chooses the problem, in which the numbers of module of the tasks are more than the number of processors of the distributed system. The model addressed in this paper is based on the consideration of processing time, cost and reliability of the module of the tasks to the various processors. The communication period amongst the module of the tasks is also used. The method is presented in algorithmic form and implemented on the several sets of input data to test the performance and effectiveness of the algorithm. As it is the common requirement for any assignment that the tasks have to be processed either with minimum time or minimum cost or maximum reliability. The example mentioned in this paper has three tasks and solved it in such a way that the task $t_{l}$ processed with minimum time, the task $t_{2}$ with minimum cost while the task $t_{3}$ with maximum reliability. The optimal results are mentioned in Table 4 of the previous section. The Table 5(a), 5(b) and 5(c) shows the optimal results as obtain after implementing the present algorithm for all three options viz. time, cost, and reliability for each and every task.

Table 5(a). Optimal results for task $t_{1}$

\begin{tabular}{|c|c|c|c|}
\hline \multirow{2}{*}{ Processor } & \multicolumn{3}{|c|}{ Assignment based on } \\
\cline { 2 - 4 } & time & Cost & reliability \\
\hline $\mathrm{p}_{1}$ & $\mathrm{~m}_{11} * \mathrm{~m}_{14}$ & $\mathrm{~m}_{13}$ & $\mathrm{~m}_{13}$ \\
\hline $\mathrm{p}_{2}$ & $\mathrm{~m}_{12} * \mathrm{~m}_{15}$ & $\mathrm{~m}_{11} * \mathrm{~m}_{14}$ & $\mathrm{~m}_{11} * \mathrm{~m}_{15}$ \\
\hline $\mathrm{p}_{3}$ & $\mathrm{~m}_{13}$ & $\mathrm{~m}_{12} * \mathrm{~m}_{15}$ & $\mathrm{~m}_{12} * \mathrm{~m}_{15}$ \\
\hline Optimal Result & 590 & 12000 & 0.996929 \\
\hline
\end{tabular}

Table 5(b). Optimal results for task $t_{2}$

\begin{tabular}{|c|c|c|c|}
\hline \multirow{2}{*}{ Processor } & \multicolumn{3}{|c|}{ Assignment based on } \\
\cline { 2 - 4 } & time & Cost & reliability \\
\hline $\mathrm{p}_{1}$ & $\mathrm{~m}_{21}$ & $\mathrm{~m}_{22} * \mathrm{~m}_{24}$ & $\mathrm{~m}_{23}$ \\
\hline $\mathrm{p}_{2}$ & $\mathrm{~m}_{22} * \mathrm{~m}_{24}$ & $\mathrm{~m}_{21}$ & $\mathrm{~m}_{21}$ \\
\hline $\mathrm{p}_{3}$ & $\mathrm{~m}_{23}$ & $\mathrm{~m}_{23}$ & $\mathrm{~m}_{22} * \mathrm{~m}_{24}$ \\
\hline Optimal Result & 540 & 9000 & 0.998442 \\
\hline
\end{tabular}

Table 5(c). Optimal results for task $t_{3}$

\begin{tabular}{|c|c|c|c|}
\hline \multirow{2}{*}{ Processor } & \multicolumn{3}{|c|}{ Assignment based on } \\
\cline { 2 - 4 } & time & cost & reliability \\
\hline $\mathrm{p}_{1}$ & $\mathrm{~m}_{31} * \mathrm{~m}_{35}$ & $\mathrm{~m}_{32} * \mathrm{~m}_{34}$ & $\mathrm{~m}_{31} * \mathrm{~m}_{35}$ \\
\hline $\mathrm{p}_{2}$ & $\mathrm{~m}_{33} * \mathrm{~m}_{36}$ & $\mathrm{~m}_{31} * \mathrm{~m}_{35}$ & $\mathrm{~m}_{32} * \mathrm{~m}_{34}$ \\
\hline $\mathrm{p}_{3}$ & $\mathrm{~m}_{32} * \mathrm{~m}_{34}$ & $\mathrm{~m}_{33} * \mathrm{~m}_{36}$ & $\mathrm{~m}_{33} * \mathrm{~m}_{36}$ \\
\hline Optimal Result & 610 & 12100 & 0.9966876 \\
\hline
\end{tabular}

\section{TIME COMPLEXITY}

It is known that the analysis of an algorithm is mainly focuses on time complexity. Time complexity is a function of input size ' $n$ '. It is referred to as the amount of time required by an algorithm to run to completion. The time complexity of the above mentioned algorithm is $\mathrm{O}\left(\mathrm{m}^{2} \mathrm{n}^{2}\right)$. By taking several input examples, the above algorithm returns results as mentioned in Table 6

Table 6. Time Complexity

\begin{tabular}{|c|c|c|}
\hline $\begin{array}{c}\text { No. of } \\
\text { processors } \\
(\mathbf{n})\end{array}$ & $\begin{array}{c}\text { No. of tasks } \\
(\mathbf{m})\end{array}$ & $\begin{array}{c}\text { Optimal } \\
\text { Results }\end{array}$ \\
\hline 3 & 4 & 144 \\
\hline 3 & 5 & 225 \\
\hline 3 & 6 & 324 \\
\hline 3 & 7 & 441 \\
\hline 3 & 8 & 576 \\
\hline 4 & 5 & 400 \\
\hline 4 & 6 & 576 \\
\hline 4 & 7 & 784 \\
\hline 4 & 8 & 1024 \\
\hline 4 & 9 & 1296 \\
\hline 5 & 6 & 900 \\
\hline 5 & 7 & 1225 \\
\hline 5 & 8 & 1600 \\
\hline 5 & 9 & 2025 \\
\hline 5 & 10 & 2500 \\
\hline
\end{tabular}

The graphical representations of the results are shown by Fig 1,2 and 3.

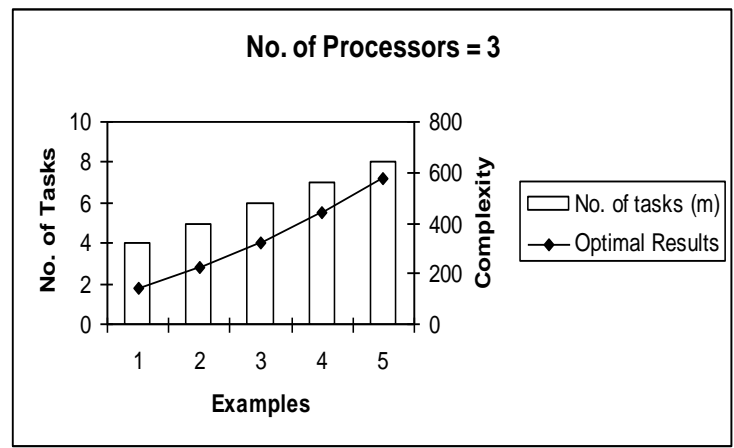

Fig 1: Graphical representation of results where $n=3$ 


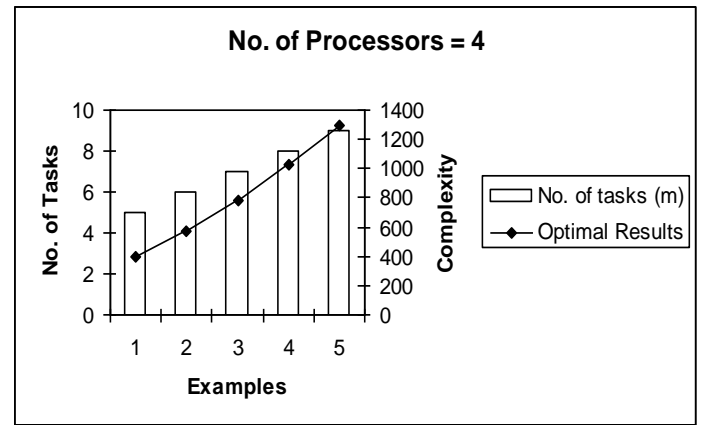

Fig 2: Graphical representation of results where $n=4$

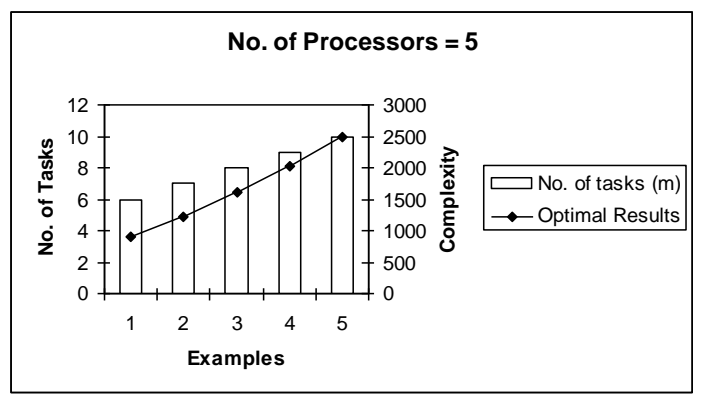

Fig 3: Graphical representation of results where $n=5$

\section{COMPLEXITY COMPARISION}

The performance of the algorithm is compared with the algorithm suggested by Richard et al (28). Following Table 7 shows the time complexity comparison between algorithm (28) with present algorithm.

Table 7. Complexity Comparison

\begin{tabular}{|c|c|c|c|}
\hline $\begin{array}{c}\text { Processors } \\
\mathbf{n}\end{array}$ & $\begin{array}{c}\text { Tasks } \\
\mathbf{m}\end{array}$ & $\begin{array}{c}\text { Time } \\
\text { Complexity } \\
\text { of } \\
\text { algorithm } \\
\mathbf{( 2 8 )} \mathbf{O}\left(\mathbf{n}^{\mathbf{m}}\right)\end{array}$ & $\begin{array}{c}\text { Time } \\
\text { Complexity } \\
\text { of present } \\
\text { algorithm } \\
\mathbf{O}\left(\mathbf{m}^{\mathbf{2}} \mathbf{n}^{\mathbf{2}}\right)\end{array}$ \\
\hline 3 & 4 & 81 & 144 \\
\hline 3 & 5 & 243 & 225 \\
\hline 3 & 6 & 729 & 324 \\
\hline 3 & 7 & 2187 & 441 \\
\hline 3 & 8 & 6561 & 576 \\
\hline 4 & 5 & 1024 & 400 \\
\hline 4 & 6 & 4096 & 576 \\
\hline 4 & 7 & 16384 & 784 \\
\hline 4 & 8 & 65536 & 1024 \\
\hline 4 & 9 & 262144 & 1296 \\
\hline 5 & 6 & 15625 & 900 \\
\hline 5 & 7 & 78125 & 1225 \\
\hline 5 & 8 & 390625 & 1600 \\
\hline 5 & 9 & 1953125 & 2025 \\
\hline 5 & 10 & 9765625 & 2500 \\
\hline
\end{tabular}

From the Table 7 it is clear that present algorithm is much better for optimal allocation of tasks that upgrade the performance of distributed system. The graphical representation of the data as mentioned in Table 7 is shown through Fig 4, 5 and 6.

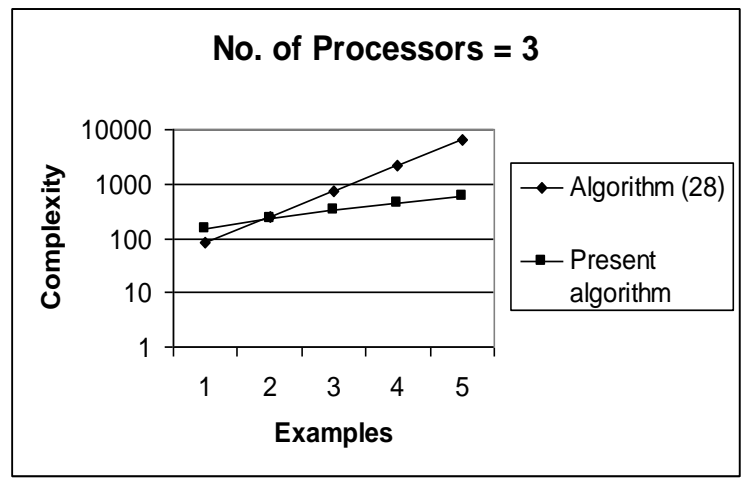

Fig 4: Graphical representation of data where $n=3$

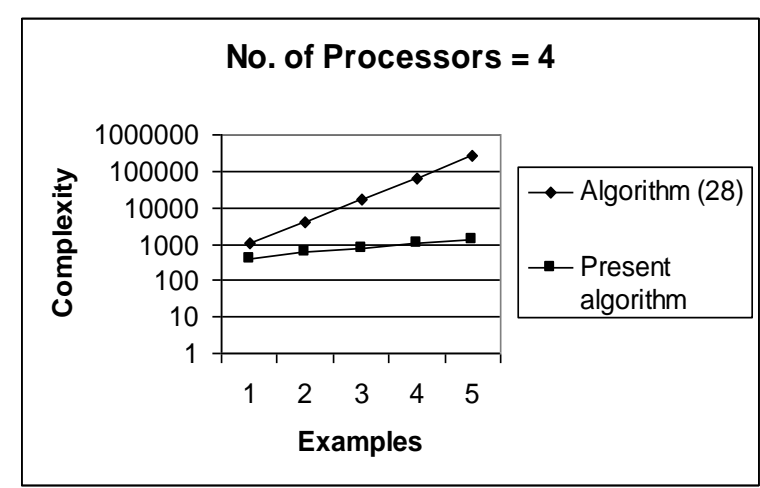

Fig 5: Graphical representation of data where $n=4$

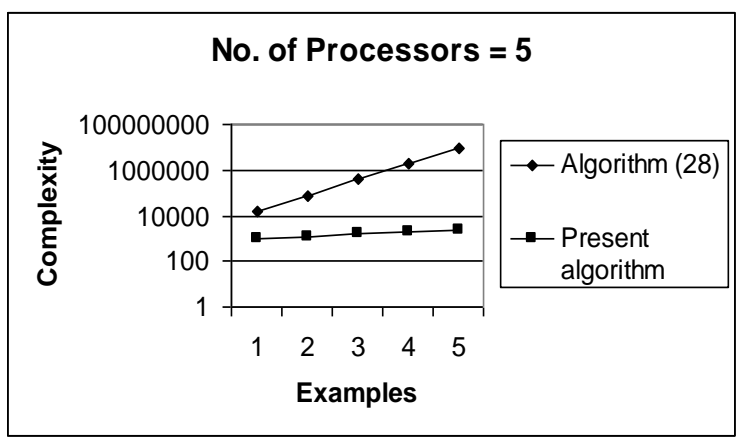

Fig 6: Graphical representation of data where $n=5$

\section{REFERENCES}

[1] Anapathur, Ramesh, V., Twigg, David W., Sandadi, Upender R. and Sharma, Tilak C. 2002. Reliability Analysis of System with Operation Time Management, IEEE Transactions on Reliability, 51, 39-48.

[2] Bahi, Jacques, Couturier, Raphaël and Vernier, Flavien. 2005. Synchronous distributed load balancing on dynamic networks, Elsevier Inc., 65(11), 1397-1405.

[3] Bierbaum, Rene L., Brown, Thomas D. and Kerschen, Thomas J. 2002, Model-Based Reliability Analysis, IEEE Transactions on Reliability, 51, 133-140.

[4] Chiu, Steve C., Liao, Wei-keng, Choudhary, Alok N. and Kandemir, Mahmut T. 2005. Processor-embedded distributed smart disks for I/O-intensive workloads: architectures, performance models and evaluation. Elsevier Inc., 65(4), 532-55.

[5] Coit, D.W. and Smith, A.E. 1996. Reliability Optimization of Series Parallel Systems using a Genetic 
Algorithm, IEEE Transactions on Reliability, 45, 254260.

[6] Contreras, Javier, Losi, Arturo, Russo, Mario and Wu, Felix F. 2000. DistOpt: A Software Framework for Modeling and Evaluating Optimization Problem Solutions in Distributed Environments", Elsevier Inc., 60(6), $741-763$.

[7] Ensink, Brian, Stanley, Joel and Adve, Vikram. 2003. Program Control Language: a programming language for adaptive distributed applications, Elsevier Inc., Vol. 63(12) $1082-1104$

[8] Fitzgerald, Kent, Latifi, Shahram and Srimani, Pradip K. 2002. Reliability Modeling and Assessment of the StarGraph Networks, IEEE Transactions on Reliability, 51, 49-57.

[9] Grosu, Daniel and Chronopoulos, Anthony T. 2005. Noncooperative load balancing in distributed systems. Elsevier Inc., 65(9), 1022-1034.

[10] Iqbal, Saeed and Carey, Graham F. 2005. Performance analysis of dynamic load balancing algorithms with variable number of processors. Elsevier Inc., 65(8), 934948

[11] Jan, Gene Eu and Lin, Ming-Bo. 2005. Concentration, load balancing, partial permutation routing, and superconcentration on cube-connected cycles parallel computers. Elsevier Inc., 65(12),1471-1482.

[12] Kandemir M., Ramanujam J. and Choudhary A. 2000. Compiler Algorithms for Optimizing Locality and Parallelism on Shared and Distributed-Memory Machines, Elsevier Inc., 60(8), 924 - 965.

[13] Kuang, Hairong, Bic, Lubomir F. and Dillencourt, Michael B. 2005. PODC: Paradigm-oriented distributed computing. Elsevier Inc., 65(4), 506-518.

[14] Kumar, Avanish. 1999. Optimizing for the Dynamic Task Allocation, in proceedings of the 'III Conference of the International Academy of Physical Sciences, 1999 Allahabad, 281-294.

[15] Kumar, Avanish. 2001. An Algorithm for Optimal Index to Tasks Allocation Based on Reliability and cost, in proceedings of 'International Conference on Mathematical Modeling' 2001Roorkee, 150-155.

[16] Kumar, V. Singh, M. P. and Yadav, P.K. 1995. An Efficient Algorithm for Allocating Tasks to Processors in a Distributed System, in proceedings of the '19th National system conference, SSI', 1995 Coimbatore, 8287.

[17] Kumar, V. Singh, M.P. and Yadav, P.K. 1995. A Fast Algorithm for Allocating Tasks in Distributed Processing System, in proceedings of the ' 30 th Annual Convention of CSI', 1995 Hyderabad, 347-358.

[18] Kumar, V. Singh, M.P. and Yadav, P.K. 1996. An Efficient Algorithm for Multi-processor Scheduling with Dynamic Reassignment, in proceedings of the ' 6 th
National seminar on theoretical Computer Science', 1996 Banasthally Vidyapeeth, 105-118.

[19] Kwok,Yu-Kwong, Maciejewski, Anthony A., Siegel,Howard Jay, Ahmad, Ishfaq and Ghafoor, Arif. 2006. A semi-static approach to mapping dynamic iterative tasks onto heterogeneous computing systems, Elsevier Inc., 66(1), 77-98.

[20] Lin, Min-Sheng 2002. A Linear-time Algorithm for Computing K-terminal Reliability on Proper Interval Graphs, IEEE Transactions on Reliability, 51, 58-62.

[21] Lyu, Michael R., Rangarajan, Sampath and Moorsel, Aad P. A. Van. 2002. Optimal Allocation of test Resources for Software Reliability growth modeling in Software Development, IEEE Transactions on Reliability, 51, 183192.

[22] Mitchell D. Theys, Howard Jay Siegel and Edwin K. P. Chong. 2001. Heuristics for Scheduling Data Requests Using Collective Communications in a Distributed Communication Network Elsevier Inc., 61(9), 1337 1366.

[23] Muhammad K. Dhodhi, Imtiaz Ahmad, Anwar Yatama and Ishfaq Ahmad 2002. An Integrated Technique for Task Matching and Scheduling onto Distributed Heterogeneous Computing Systems Elsevier Inc., 62( 9), $1338-1361$.

[24] Ormon, Stephen W., Cassady, C. Richard and Greenwood, Allen G. 2002. Reliability Prediction model to Support Conceptual Design, IEEE Transactions on Reliability, 51, 151-157.

[25] Painton, L. and Campbell, J. 1992. Genetic Algorithm in Optimization of System Reliability, IEEE Transactions on Reliability, 44, 172-178.

[26] Palmer, J. and Mitrani, I. 2005. Optimal and heuristic policies for dynamic server allocation, Elsevier Inc., 65(10), 1204-1211.

[27] Ravindran, Binoy, Devarasetty, Ravi K. and Shirazi, Behrooz. 2002. Adaptive Resource Management Algorithms for Periodic Tasks in Dynamic Real-Time Distributed Systems, Elsevier Inc., 62(10) 1527 - 1547.

[28] Richard R.Y., Lee, E.Y.S. and Tsuchiya, M. 1982. A Task Allocation Model for Distributed Computer System, IEEE Transactions on Computer, 31, 41-47.

[29] Singh, M.P., Kumar, V. and Kumar, A. 1999. An Efficient Algorithm for Optimizing Reliability Index in Tasks-Allocation, Acta Ciencia Indica, xxv(m), 437-444.

[30] Ucar, Bora, Aykanat, Cevdet, Kaya, Kamer and Ikinci, Murat. 2006. Task assignment in heterogeneous computing systems. Elsevier Inc., 66(1),32-46.

[31] Wong, Han Min, Bharadwaj, Veeravalli and Gerassimos, Barlas. 2005. Design and performance evaluation of load distribution strategies for multiple divisible loads on heterogeneous linear daisy chain networks. Elsevier Inc., 65(12), 1558-1577. 